\title{
DHX29 and elF3 cooperate in ribosomal scanning on structured mRNAs during translation initiation
}

\author{
VERA P. PISAREVA and ANDREY V. PISAREV \\ Department of Cell Biology, SUNY Downstate Medical Center, Brooklyn, New York 11203, USA
}

\begin{abstract}
Eukaryotic translation initiation is a complex process involving many components. elF3 is a scaffold for multiple initiation factors and plays multiple roles in initiation, and DHX29 helicase enhances the formation of the $48 S$ initiation complex on structured mRNAs. Because DHX29 is not a processive helicase, the mechanism underlying its activity is unclear. Here, we show that DHX29 establishes many points of contact with elF3. In particular, the unique $\mathbf{N}$ terminus of DHX29 associates with the RNA recognition motif of elF3b and the $C$ terminus of the eIF3a subunits of eIF 3 , and the disruption of either contact impairs DHX29 activity. In turn, DHX29 has weak points of contact with mRNA in the 48S initiation complex, and the pathway taken by mRNA remains unchanged. These results exclude the direct role for this protein in unwinding. Thus, DHX29 and eIF3 cooperate in scanning on structured mRNAs. Our findings support previous genetic data on the role of elF3 during scanning.
\end{abstract}

Keywords: DHX29; eIF3; ribosomal scanning; translation control; translation initiation

\section{INTRODUCTION}

Eukaryotic translation consists of four stages: initiation, elongation, termination, and ribosomal recycling. The first step, translation initiation, is the most complex and tightly regulated, and it begins with the assembly of eIF2, aminoacylated initiator tRNA (Met-tRNA ${ }_{i}{ }^{\text {Met }}$ ), and GTP into the ternary complex (TC). Subsequently, the TC and eIF5, eIF3, eIF1, and IF1A cooperatively bind to the $40 \mathrm{~S}$ subunit through the formation of the $43 S$ preinitiation complex (43S PIC). The $43 \mathrm{~S}$ PIC then loads onto the preliminary unwound $5^{\prime}$ end of mRNA via eIF4A, eIF4B, and eIF4F. After attachment, the 43S PIC scans the $5^{\prime}$-untranslated region ( $5^{\prime}$-UTR) for the initiation codon, where it stops and forms the $48 \mathrm{~S}$ initiation complex (48S IC), thereby establishing the codon-anticodon interaction in the P-site of the $40 \mathrm{~S}$ subunit. Finally, the $48 \mathrm{~S}$ IC, with the help of eIF5 and eIF5B, binds to the 60S subunit to form the $80 \mathrm{~S}$ initiation complex and initiate elongation (Jackson et al. 2010; Hinnebusch and Lorsch 2012; Asano 2014).

Efficient translation initiation on mRNAs with structured 5'-UTRs, which are more prevalent in higher eukaryotes, requires the specific DExH-box helicase DHX29 in vitro and in vivo (Pisareva et al. 2008; Parsyan et al. 2009). DHX29 depletion is also shown to impede cancer cell growth in culture and

Corresponding authors: andrey.pisarev@downstate.edu, vera.pisarev@ downstate.edu

Article published online ahead of print. Article and publication date are at http://www.rnajournal.org/cgi/doi/10.1261/rna.057851.116. in xenografts, which suggests a role for this protein in tumorigenesis (Parsyan et al. 2009). Until the recent discovery of mammalian-specific DHX29, eIF4A was the only reported helicase with a confirmed role in canonical eukaryotic translation initiation. Importantly, the mechanisms underlying the activities of eIF4A and DHX29 in initiation are different. eIF4A strictly requires accessory factors eIF4G and eIF4B, whereas DHX29 requires the 40S ribosomal subunit, which stimulates its ATPase activity (Pisareva et al. 2008; Harms et al. 2014). Because DHX29 is necessary for translation during normal development and in tumorigenesis, it is critically important to understand the unique mechanism of its activity.

DHX29 consists of a unique $\mathrm{N}$ terminus, a central helicase domain, and a C-terminal region containing helicase-associated 2 (HA2) and OB domains. Recent CryoEM data have revealed the position of DHX29 in the mammalian reconstituted 43S PIC (Hashem et al. 2013; des Georges et al. 2015). The main density modeled as the combination of the central helicase domain and C terminus of DHX29 is located around the tip of helix H16 of the 40 S subunit, whereas the distal part extends along the mRNA entry channel and enters the intersubunit interface up to the eIF1A-binding

(C) 2016 Pisareva and Pisarev This article is distributed exclusively by the RNA Society for the first 12 months after the full-issue publication date (see http://rnajournal.cshlp.org/site/misc/terms.xhtml). After 12 months, it is available under a Creative Commons License (Attribution-NonCommercial 4.0 International), as described at http://creativecommons.org/licenses/ by-nc/4.0\%. 
site (Hashem et al. 2013). Moreover, the main density of DHX29 establishes points of contact with the eIF3b and eIF3i subunits of eIF3 (des Georges et al. 2015). Because DHX29 is not a processive helicase, the mechanism of its activity in ribosomal scanning remains obscure, and it may either unwind the mRNA secondary structure before the latter enters the mRNA entry channel or cause the remodeling of ribosomal complexes.

Recent genetic data have revealed that eIF3 also plays an important role in ribosomal scanning (Chiu et al. 2010; Cuchalová et al. 2010; Elantak et al. 2010). Mammalian eIF3 is the largest translation factor at $\sim 800 \mathrm{kDa}$ and consists of 13 subunits $(\mathrm{a}-\mathrm{m})$. Six subunits $(\mathrm{a}, \mathrm{c}, \mathrm{e}, \mathrm{k}, \mathrm{l}$, and $\mathrm{m}$ ) contain PCI domains, whereas two subunits ( $f$ and $h$ ) contain MPN domains (Asano et al. 1997; Pena et al. 2007; Enchev et al. 2010). The PCI/MPN domains together form the stable octameric structural core of eIF3. CryoEM data on the reconstituted mammalian 43S PIC have demonstrated that this core has a five-lobed shape and sits at the solvent side of the 40S subunit opposite the platform (Hashem et al. 2013; des Georges et al. 2015). Five of the remaining eIF3 subunits are associated with the octameric core in a flexible manner. eIF3b, eIF3i, and eIF3g are combined in a separate cluster located at the mRNA entrance and connected to the core through the carboxy-terminal domain of eIF3a, whereas eIF3d is positioned at the mRNA exit behind RACK1 (des Georges et al. 2015). eIF3j, a loosely associated subunit of eIF3, has previously been shown to bind below the beak of the $40 \mathrm{~S}$ subunit (Aylett et al. 2015). This complex organization of eIF3 explains its role in almost all steps of initiation, including ribosomal subunit anti-association activity, TC recruitment to the 40S subunit, 43S PIC loading onto mRNA, and ribosomal scanning. eIF3 also acts as a protein scaffold for the assembly of the initiation complex (Jackson et al. 2010). Interestingly, Saccharomyces cerevisiae and related yeasts only have six subunits: two PCI (a and c) and four non-core (b, i, g, and j) subunits (Asano et al. 1998; Phan et al. 1998; Valásek et al. 2001).

We investigate mammalian translation by reconstituting it in vitro from individual purified and characterized components. To elucidate the mechanism of DHX29's activity in scanning, we used the reconstitution approach in combination with the UV-cross-linking technique as well as the GST pull-down assay and mutational analysis. We found that upon ribosomal binding, DHX29 could establish points of contact with eIF3 in the 48S IC. The disruption of such points of contact in the unique N terminus of DHX29 impairs the protein's activity during scanning. Concurrently, DHX29 produces weak points of contact with mRNA in initiation complexes, and the pathway taken by the mRNA in the presence of DHX29 remains the same. Therefore, DHX29 affects scanning by remodeling ribosomal complexes. In this study, the novel functional interaction during translation between DHX29 and a canonical initiation factor is shown. Our findings also support the genetic data on eIF3's function during scanning.

\section{RESULTS}

\section{DHX29 does not establish strong contacts with mRNA in the $48 \mathrm{~S} \mathrm{IC}$ as revealed by UV-cross-linking experiments}

DHX29 in the 43S PIC is located around the tip of helix H16 near the mRNA entrance (Pisareva et al. 2008; Hashem et al. 2013). Although the position of DHX29 in the context of the 43S PIC is determined, mRNA is absent from this complex, and its path in the presence of DHX29 is unknown. It is assumed that the mechanism underlying the unwinding activity of RNA helicases involves the mRNA passing through and making contact with the active center of the helicase domain (Linder and Jankowsky 2011). DHX29 was demonstrated to have weak RNA duplex-unwinding activity in vitro (Pisareva et al. 2008). Therefore, to discriminate between direct unwinding and ribosomal complex remodeling by DHX29 during scanning, it is critical to determine whether the protein makes contacts with the mRNA in the mRNA entry channel of DHX29-associated initiation complex.

To obtain insights into the mechanism of DHX29's activity, we assembled the $48 \mathrm{~S}$ IC on a (CAA $)_{n}$-repeat-based single-stranded model mRNA with a centrally positioned AUG codon and a locally introduced unique uridine. We prepared a set of radiolabeled model mRNAs with a 4-thiouridine (4-thioU) in one of the following positions: $+5,+7,+9$, +11 , +13 , and +15 (Fig. 1A). In addition to containing 4thioU at the indicated unique position, all of the mRNA fragments contain 4-thioU in the AUG codon. Such model mRNAs efficiently form 48S ICs in the reconstituted system. The position of a toeprint signal at +17 nt from AUG unambiguously indicates that the $48 \mathrm{~S}$ IC is assembled on the AUG start codon of 4 -thioU-containing “ +13 ” and “ +15 " mRNAs regardless of the presence of DHX29 (Fig. 1B).

In the control experiment, we evaluated cross-linking patterns for the 48S ICs assembled in the absence of DHX29. mRNA specifically cross-links to uS19 at the +5 position and to uS3/uS5 at the $+9,+11$, and +13 positions (with the peak intensity at +11 ) (Fig. 1C, lanes $1,5,7,9$ ), as previously reported (Pisarev et al. 2008). The addition of DHX29 to the canonical factors in the system resulted in its weak cross-linking to all of the tested mRNA bases (Fig. 1C). Although DHX29 is located in the 43S PIC along the mRNA entry channel, such a broad nucleotide interval of cross-linking suggests a nonspecific rather than specific signal. In either case, the low efficiency of cross-linking to DHX29 indicates that mRNA in the mRNA entry channel does not pass through the helicase. Consistently, the pattern of the cross-linked ribosomal proteins in the presence of DHX29 remains the same (Fig. 1C).

Notably, in the presence of DHX29, we observed an increase of cross-linking signals at all positions, which we hypothetically assigned to eIF3 subunits (Fig. 1C). The increase of these cross-linking signals could result from the partial 
A
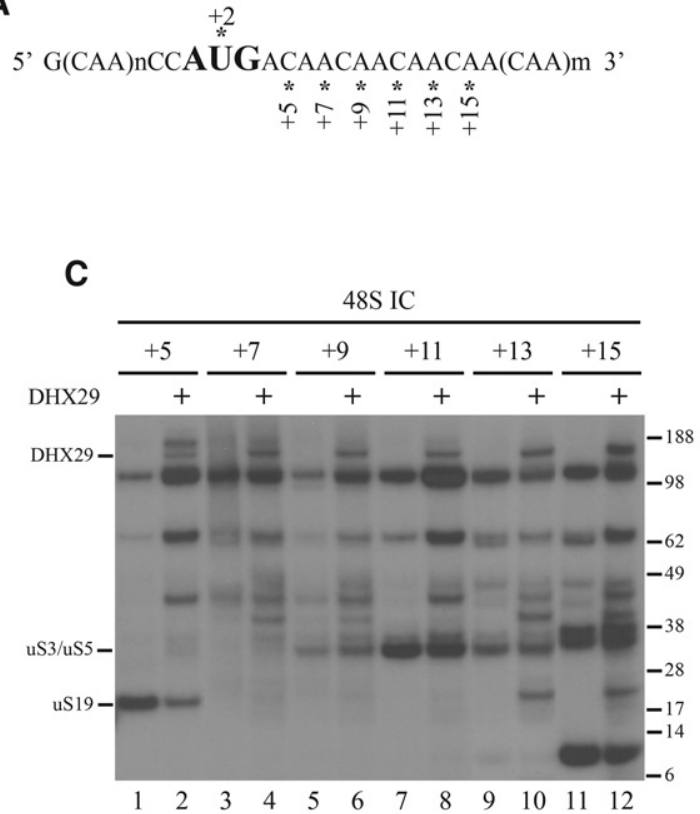

B

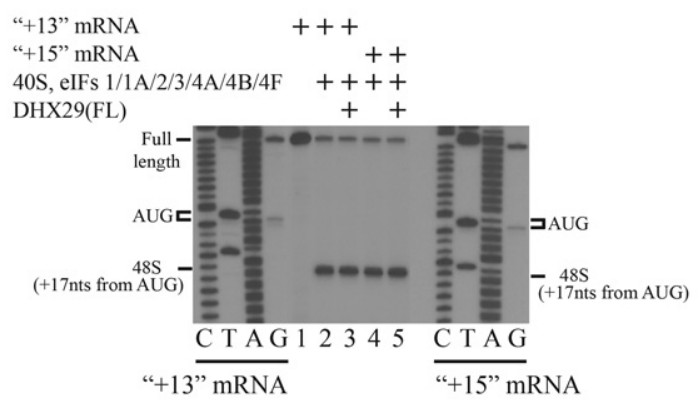

D

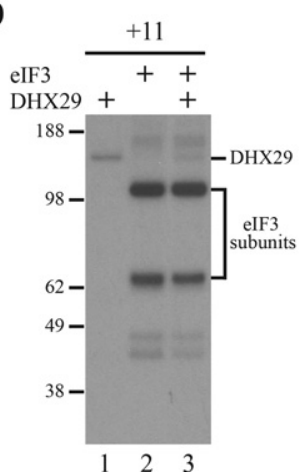

FIGURE 1. Influence of DHX29 on mRNA cross-linking in the mRNA entry channel of the 48S IC. (A) Structure of mRNAs utilized for the UVcross-linking assay. The positions of unique uridines relative to the AUG codon are indicated by asterisks below the sequence. $(B)$ Toeprint analysis of the formation of the 48 S IC on " +13 " and " +15 " mRNAs containing 4 -thioU. The initiation codon and position of the assembled 48 S IC are indicated.

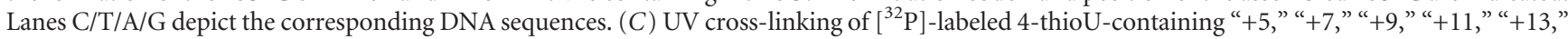
and " +15 " mRNAs with components of the 48S IC assembled in the presence and absence of DHX29 assayed by SDS-PAGE and autoradiography. Positions of the cross-linked proteins and molecular-weight markers are indicated. $(D)$ UV-cross-linking of $\left[{ }^{32} \mathrm{P}\right]$-labeled " +11 ” mRNA containing 4-thioU with eIF3 and DHX29 in the minimal system tested by SDS-PAGE and autoradiography. Positions of cross-linked proteins and molecularweight markers are indicated.

spontaneous dissociation of $48 \mathrm{~S}$ ICs with the subsequent eIF3/mRNA complex formation during UV-irradiation assay. Therefore, we also evaluated the effect of DHX29 on cross-linking in the eIF3/mRNA binary system. In the absence of DHX29, “+11" mRNA strongly cross-links to eIF3 subunits (Fig. 1D, lane 2). DHX29 alone, in the amount equimolar to eIF3, cross-links to mRNA inefficiently, indicating the weak RNA-binding capacity of this protein (Fig. 1D, lane 1). The addition of DHX29 does not affect the intensity of eIF3 cross-linking signals (Fig. 1D, lane 3 ).

Considering all of the data, we conclude that DHX29 does not establish strong contacts with the mRNA in the mRNA entry channel of $48 \mathrm{~S}$ IC. Importantly, the pattern of cross-linking ribosomal proteins (uS3/uS5 and uS19) does not change, suggesting that the mRNA path in the presence of DHX29 remains the same. Nevertheless, we discovered the specific changes in cross-linking $(180 \mathrm{kDa}$ protein at the +5 position and $20 \mathrm{kDa}$ protein at the +13 and +15 positions) induced by DHX29, favoring the hypothesis that DHX29 acts by remodeling ribosomal complexes rather than by directly unwinding the mRNA secondary structure.

\section{DHX29 establishes multiple contacts with eIF3}

Based on the CryoEM structure of DHX29-associated 43S PIC, DHX29 establishes contacts with the eIF3b and eIF3i subunits of eIF3. Using a biochemical approach, we recently reported that DHX29 also makes a contact with eIF1A, but the disruption of this contact does not affect DHX29-mediated mRNA secondary structure unwinding during scanning (Pisareva and Pisarev 2016). Therefore, we hypothesized that a suggested rearrangement of ribosomal complexes could be a result of the direct interaction between DHX29 and EIF3 in initiation complexes. To test our hypothesis, we used a GST pull-down assay and prepared the GST-tagged full-length form of DHX29 and its C-terminal deletion mutants: DHX29 (1-1039) containing an $\mathrm{N}$ terminus and a helicase domain, and DHX29 (1-594) containing only the N terminus (Fig. 2A). After the expression in E. coli, the GST-tagged proteins were prebound from the lysate to Glutathione Sepharose, incubated with purified native eIF3, resolved using SDS-PAGE, and analyzed by immunoblotting. The GST pull-down assay revealed that eIF3 binds to all of the tested forms of DHX29 but not to the GST control protein (Fig. 

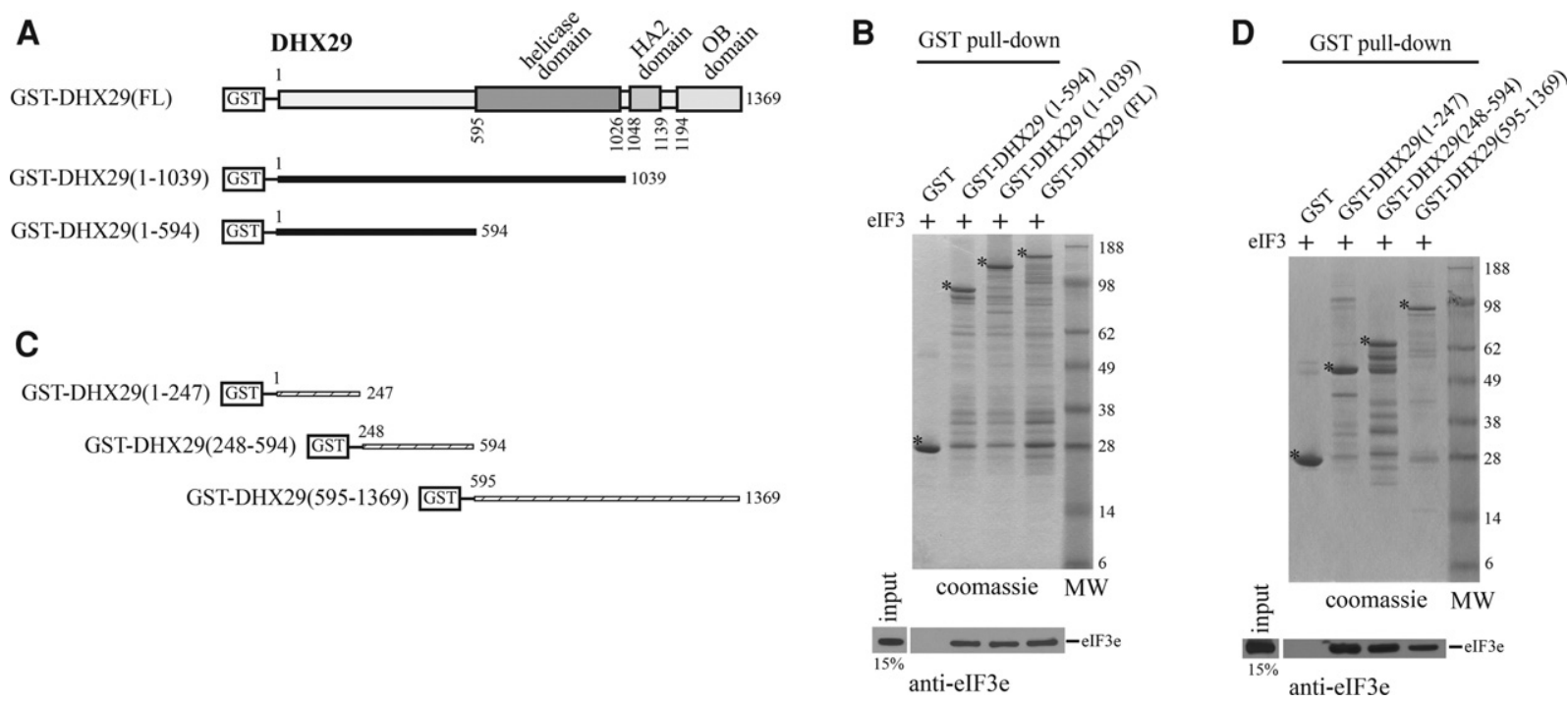

FIGURE 2. Interaction between DHX29 and eIF3. $(A, C)$ GST-tagged DHX29 and fragments used in the GST pull-down assay. (B,D) Binding of different fragments of DHX29 to eIF3 analyzed by GST pull-down assay. Each binding reaction after elution from glutathione resin along with a $15 \%$ input amount of the corresponding reaction mixture was assayed by SDS-PAGE and either Coomassie blue stained or immunoblotted. The asterisks indicate the main bands of the GST-tagged fragments. Positions of the molecular-weight markers are shown.

2B). We discovered the novel contact between DHX29 (1-594), containing only the unique $\mathrm{N}$ terminus, and eIF3. To locate this contact more precisely, we prepared another set of GST-tagged DHX29 mutants: DHX29 (1-247), containing the first half of the N terminus; DHX29 (248-594), containing the second half of the N terminus; and DHX29 (595-1369), containing the helicase domain and C terminus (Fig. 2C). All three DHX29 mutants establish contacts with eIF3 as revealed by a GST pull-down assay (Fig. 2D). The detected interaction between DHX29 (595-1369) and eIF3 is attributable to contacts with eIF3b and eIF3i, as previously reported (des Georges et al. 2015). Therefore, we did not characterize this part of the helicase. Notably, DHX29 contains binding sites in both halves of its unique $\mathrm{N}$ terminus, suggesting an important role for the $\mathrm{N}$ terminus in initiation.

We aimed to identify the eIF3 subunits responsible for the interaction with the DHX29 N terminus. Considering the CryoEM data on the DHX29-bound 43S PIC, we suggested that the N terminus of DHX29 interacts with eIF3b. To test this hypothesis, we utilized GST-tagged murine eIF3b and deletion mutants representing different protein domains (Fig. 3A). We found that DHX29 binds to eIF3b but not to the GST control protein and that the RNA recognition motif (RRM) in the $\mathrm{N}$ terminus of eIF3b is the shortest region interacting with DHX29 (Fig. 3B). To localize the site in DHX29 that contacts the eIF3b RRM, we purified a recombinant deletion mutant eIF3b (1-300) (Fig. 3C) that included an RRM and assayed its interaction with GST-tagged DHX29 (1-247), DHX29 (248-594), and DHX29 (5951369). eIF3b (1-300) bound to DHX29 (248-594), which supports our hypothesis that eIF3b is responsible for the interaction with the DHX29 N terminus (Fig. 3D).
To identify the binding partner of DHX29 (1-247), we again referred to CryoEM data (Hashem et al. 2013; des Georges et al. 2015). Taking into account the superposition of DHX29 and eIF3 in the DHX29-associated 43S PIC, we hypothesized that the first half of DHX29's N terminus contacts the $\mathrm{C}$ terminus of eIF3a. To test our hypothesis, we prepared two GST-tagged human eIF3a deletion mutants: eIF3a (1-782), containing the N-terminal half and eIF3a (7831382), containing the C-terminal half (Fig. 3E). We also cloned and expressed DHX29 (1-247), containing the first half of the DHX29 N terminus. As expected, DHX29 (1-247) bound efficiently to eIF3a (783-1382) (Fig. 3F). In conclusion, DHX29 establishes multiple contacts with eIF3. The first half of the DHX29 N terminus binds to the C-terminal half of eIF3a, whereas the second half of the DHX29 N terminus establishes contact with the eIF3b RRM.

\section{Contacts between the DHX29 $\mathrm{N}$ terminus and eIF3a and elF3b subunits are essential for unwinding the mRNA secondary structure during ribosomal scanning}

To determine whether contacts between DHX29 and IF3 are important for the activity of helicase during scanning, we used a mutational analysis coupled with the functional testing of mutants in the reconstituted system. We started our study with contacts detected in the DHX29 N terminus. A set of His-tagged N-terminal deletion mutants of DHX29 (DHX29 [25-1369], DHX29 [54-1369], DHX29 [911369], DHX29 [125-1369], DHX29 [248-1369], DHX29 [377-1369], DHX29 [448-1369], and DHX29 [509-1369]) was constructed for expression in E. coli. Only DHX29 (251369), DHX29 (54-1369), and DHX29 (248-1369) were 

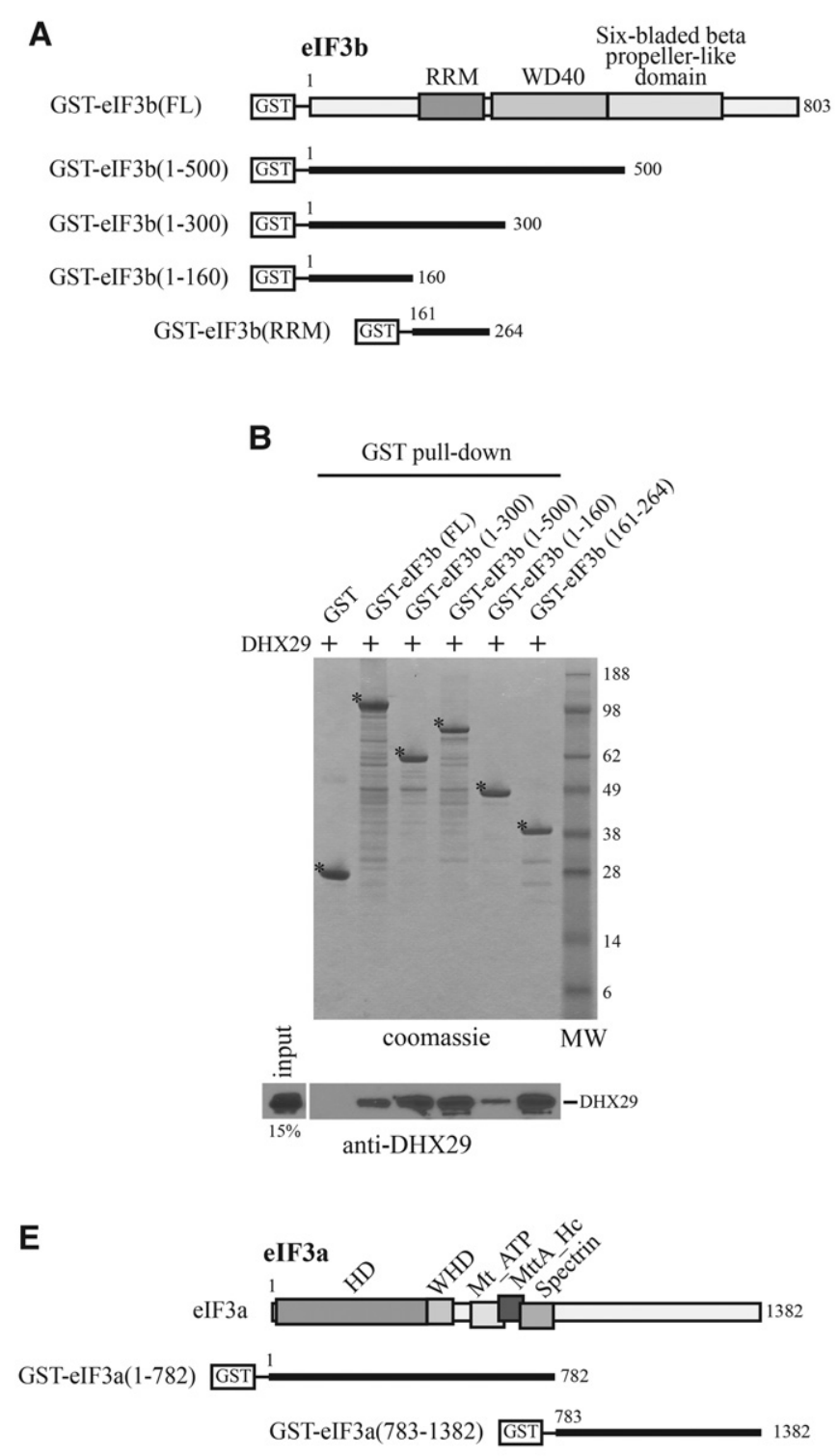

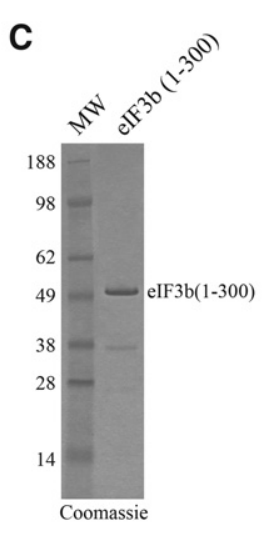

D

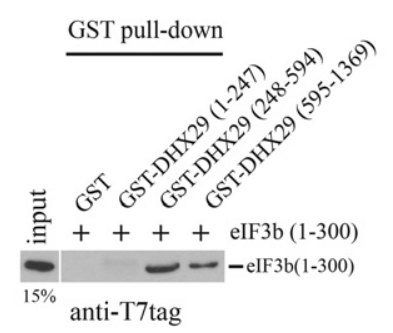

$\mathbf{F}$

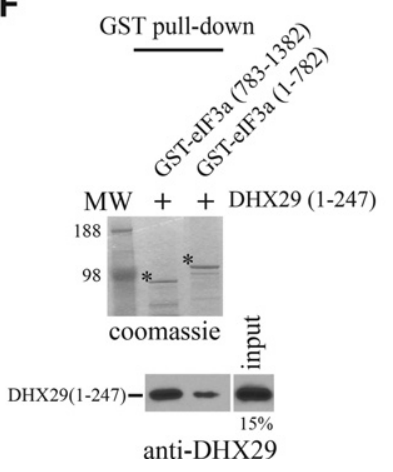

FIGURE 3. Association of DHX29 with the eIF3a and eIF3b subunits of eIF3. ( $A, E$ ) Organization of GST-tagged $(A)$ eIF3b and its fragments, and $(E)$ different parts of eIF3a used in the GST pull-down assay. $(B, D, F)$ Association of different fragments of $(B)$ eIF3b with DHX29, $(D)$ DHX29 with eIF3b (1-300), and (F) eIF3a with DHX29 (1-247) examined by a GST pull-down assay. Each binding reaction after elution from glutathione resin along with 15\% input amount of the corresponding reaction mixture were assayed by SDS-PAGE and either Coomassie blue staining or immunoblotting. The asterisks indicate the main bands of the GST-tagged fragments. Positions of the molecular-weight markers are shown. $(C)$ Purified recombinant eIF3b (1-300) resolved by SDS-PAGE.

soluble after expression and subsequently purified (Fig. 4A, B). To test the functional activity of the mutants in the system, we utilized (AUG at -6)-Stem mRNA, which was previously described (Abaeva et al. 2011). This mRNA contained the single-stranded region of (CAA) repeats with a centrally introduced 9 bp hairpin and three AUG codons (Fig. 4C). The first AUG (AUG1) was inserted upstream of the hairpin (A in AUG1 is at the "-6" position from a hairpin); the second AUG (AUG2) was in the loop of the hairpin; and the third AUG (AUG3) was located downstream from the hairpin. AUG3 was followed by the $\beta$-glucuroni- dase ORF. All of the AUGs were in an optimal nucleotide context.

In the absence of DHX29, the 48S IC is assembled on (AUG at -6)-Stem mRNA exclusively at AUG1 (Fig. 4D, lanes 1,2). Because the hairpin enters the mRNA binding channel of the $40 \mathrm{~S}$ subunit in an intact unwound state, the 48S IC yields a toeprint signal at a position +11-12 nt after the hairpin. The presence of DHX29 results in efficient unwinding of the hairpin and placing it in the single-stranded state in the mRNA-binding channel of the $40 \mathrm{~S}$ subunit yielding the toeprint signal at a common position $+16-17 \mathrm{nt}$ from AUG1 


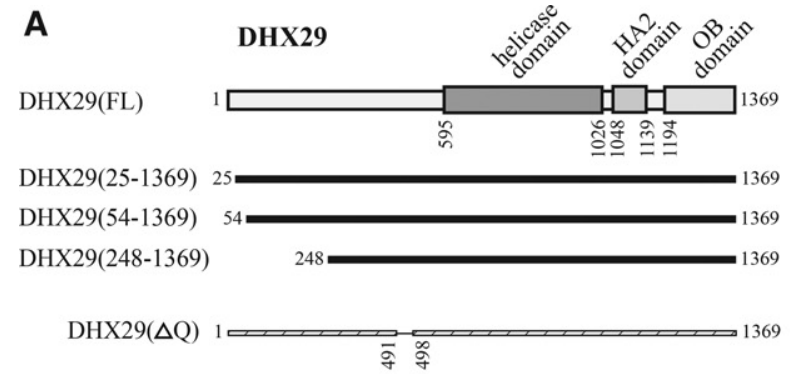

B

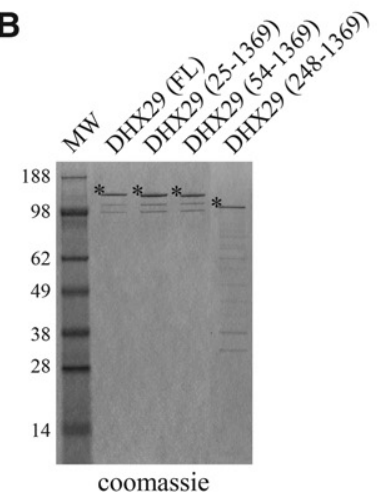

C

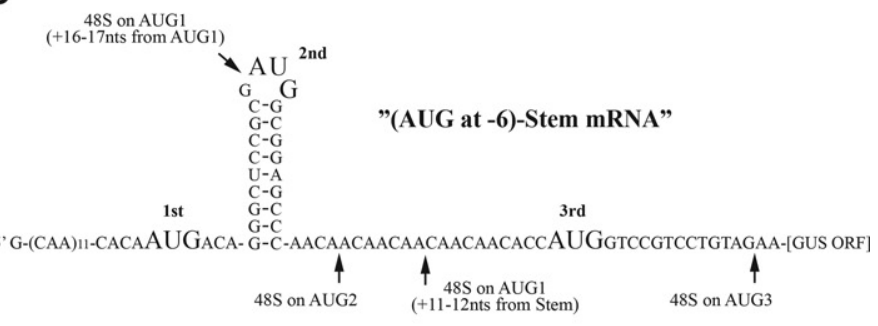

D

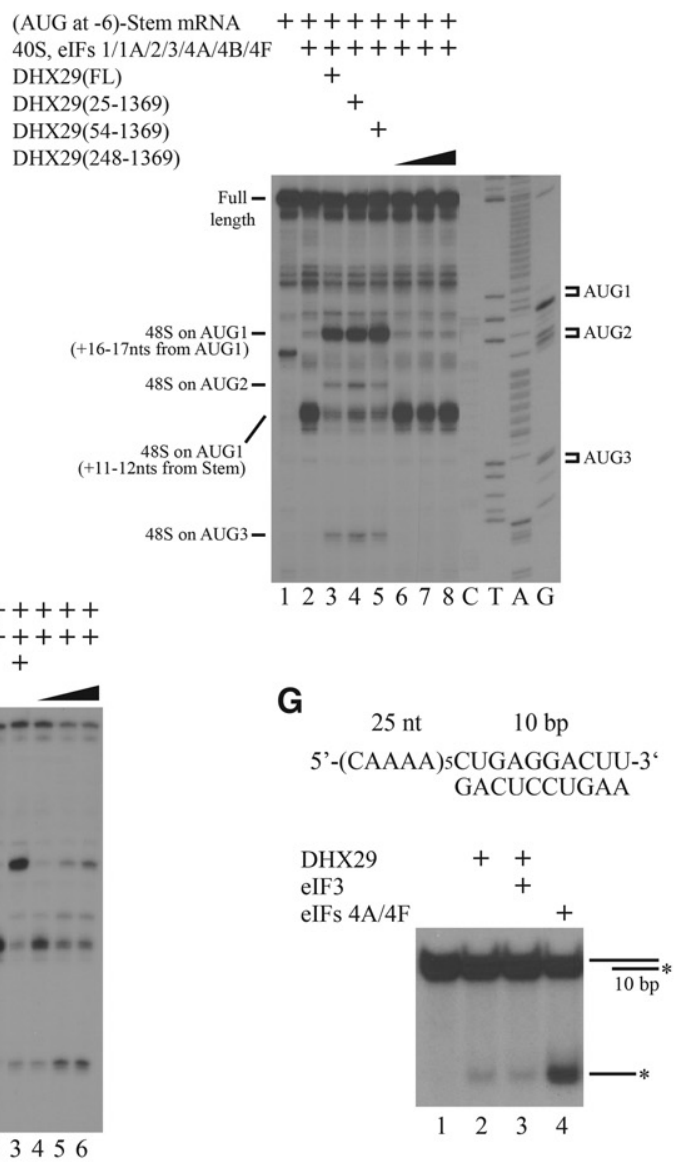

FIGURE 4. Role of contacts between DHX29 and eIF3 in the DHX29-mediated unwinding of mRNA secondary structure during ribosomal scanning. (A) Organization of deletion mutants of DHX29. (B) Purified deletion mutants of DHX29 resolved by SDS-PAGE. The asterisks indicate the main bands of the proteins. (C) Structure of (AUG at -6)-Stem mRNA. The positions of the toeprint signals for the 48S ICs assembled on different start codons are indicated. $(D, F)$ Toeprint analysis of the formation of the $48 \mathrm{~S}$ IC on (AUG at -6$)$-Stem mRNA in the presence of different DHX29 mutants. Initiation codons and positions of the assembled 48S IC are indicated. Lanes C/T/A/G depict the corresponding DNA sequence. (E) Binding of DHX29 (FL) and DHX29 $(\Delta \mathrm{Q})$ to eIF3b (1-300) tested by GST pull-down assay. Each binding reaction after elution from glutathione resin was assayed together with 15\% input amounts of both DHX29 forms by SDS-PAGE and immunoblotting with anti-DHX29 antibodies. Comparison of the input DHX29 (FL) and DHX29 $(\Delta \mathrm{Q})$ was analyzed by SDS-PAGE followed by Coomassie blue staining. Positions of both DHX29 forms as well as the molecular-weight markers are shown. $(G)$ Native PAGE representing the unwinding of 10 bp RNA duplex with a 25nt-long overhanging $5^{\prime}$-end by DHX29 alone, DHX29/eIF3, and eIF4A/eIF4F. Positions of the $\left[{ }^{32} \mathrm{P}\right]$-labeled duplex and single-stranded RNAs are indicated.

(Fig. 4D, lane 3), as previously reported (Abaeva et al. 2011). The addition of DHX29 (25-1369) or DHX29 (54-1369) shifts the toeprint signal as efficiently as DHX29 (FL) (Fig. $4 \mathrm{D}$, lanes 4,5). However, the presence of DHX29 (2481369 ) at different concentrations does not change the position of the toeprint signal (Fig. 4D, lanes 6-8). Therefore, the first half of the DHX29 N terminus is essential for the activity of the protein, suggesting the critical importance of the detected contact between DHX29 and eIF3a during scanning on structured mRNAs. 
To investigate the role of the contact between the second half of the DHX29 N terminus and eIF3b RRM, we analyzed the DHX29 sequence and detected the polyglutamine (polyQ) motif, which consisted of eight consecutive glutamines and was located at a position from 491 to 498 . polyQ motifs are shown to be important for protein-protein interactions (Schaefer et al. 2012). We hypothesized that the polyQ motif of DHX29 could be responsible for the interaction with eIF3b. To test our hypothesis, we constructed a Histagged DHX29 mutant with a deleted polyQ motif (DHX29 $[\Delta \mathrm{Q}])$ for E. coli expression and purification (Fig. 4A). In a GST pull-down assay, DHX29 $(\Delta \mathrm{Q})$ binding to GST-tagged eIF3b (1-300) containing the RRM motif was less efficient compared with that of DHX29 (FL) (Fig. 4E). Moreover, in the reconstituted system, DHX29 $(\Delta \mathrm{Q})$ stimulates only a moderate mRNA secondary structure unwinding during the 48S IC assembly on (AUG at -6)-Stem mRNA compared with that of DHX29 (FL), as revealed by the position of toeprint signals (Fig. 4F). Therefore, the interaction between the second half of the DHX29 N terminus and eIF3b RRM is important for DHX29's activity during ribosomal scanning on structured mRNAs.

DHX29 alone is a weak helicase, and it can unwind only small stem-loop duplexes (Pisareva et al. 2008). We tested whether eIF3 stimulates the helicase activity of DHX29 via a direct unwinding assay based on thin-layer chromatography (TLC). We used a 10-nt duplex with a 25 -nt $5^{\prime}$-overhanging extension, which was described in a DHX29-duplexunwinding study (Pisareva et al. 2008). As a result, eIF3 does not stimulate the weak duplex-unwinding activity of DHX29, whereas eIF4A and eIF4F control proteins efficiently unwind this duplex (Fig. 4G).

\section{C terminus of DHX29 is sufficient and $\mathrm{N}$ terminus is dispensable for the ribosomal binding of protein, and $O B$ domain at the end of the $C$ terminus is critical for this association}

CryoEM data on DHX29-associated 43S PIC have revealed the presence of contacts between the DHX29 density, which is modeled as the helicase domain and C terminus, and the 40S subunit (Hashem et al. 2013). Previous biochemical studies have described the additional ribosomal binding site located at DHX29's N terminus (Dhote et al. 2012). Ribosomal binding of DHX29 is essential for its ATPase as well as its helicase activities (Pisareva et al. 2008). Considering the dependence of DHX29 activity on the ribosomal association, the $40 \mathrm{~S}$ subunit-binding sites are an important determinant of DHX29 functionality. In our experiments, $\operatorname{DHX} 29(\Delta \mathrm{Q})$ in the reconstituted system was not as active as DHX29 (FL), and DHX29 (248-1369) was completely inactive (Fig. 4D,F). Therefore, to test whether this impaired activity was the result of disrupted ribosomal association, we studied the role of different parts of DHX29 in ribosomal binding.
We constructed a set of His-tagged deletion mutants of DHX29 N terminus (1-594) (Fig. 5A), C terminus (9831369), and an entire protein lacking the OB domain (11227). Unfortunately, DHX29 (983-1369) and DHX29 (11227) were soluble after E. coli expression in limited amounts and were not isolated. To test the importance of the DHX29 $\mathrm{N}$ terminus in ribosomal binding, a 43S PIC was reconstituted in the presence of DHX29 (1-594), purified through SDG centrifugation, run on SDS-PAGE, and analyzed by Coomassie staining and immunoblotting. DHX29 (1-594) was not found to associate with the 43S PIC, which indicates that the $\mathrm{N}$ terminus of DHX29 is dispensable for ribosomal binding (Fig. 5B). To avoid the purification step for the mutants that were soluble in limited amounts after expression, we introduced a PKA site in these constructs (Fig. 5C). This approach permitted us to obtain specifically phosphorylated mutants in the form of crude Ni-NTA eluates. The ribosomal binding protocol was modified accordingly, and the reconstituted 43S PIC was supplemented with the corresponding crude Ni-NTA eluate rather than with the purified protein. We found that PKA-DHX29 (FL) and PKA-DHX29 (983-1369) but not PKA-DHX29 (1-1227) bind to the 43S PIC, which suggests that the C terminus of DHX29 alone is sufficient for ribosomal binding and the $\mathrm{OB}$ domain at the end of the $\mathrm{C}$ terminus is critically important for this association (Fig. 5D).

Our findings contradict previously published biochemical data that indicated that DHX29 contains two ribosomal binding sites: in the $\mathrm{N}$ terminus and at the beginning of the C terminus (Dhote et al. 2012). To investigate the role of the DHX29 C terminus in mediating the unwinding of the mRNA secondary structure during scanning, we constructed a His-tagged deletion mutant of DHX29 that lacked almost the entire C terminus (DHX29 (1-1039) for E. coli expression and purification (Fig. 5E,F). Compared with the DHX29 (FL) system, the addition of DHX29 (1-1039) at different concentrations does not promote a shift in the toeprint signal in the reconstituted system (Fig. 5G). This result supports our finding that the OB domain located at the very end of the DHX29 $\mathrm{C}$ terminus is critical for ribosomal binding and, consequently, for DHX29's activity in ribosomal scanning. Moreover, the importance of the $\mathrm{OB}$ domain for the ribosomal association and, thus, for the activity of the helicase in ribosomal scanning correlates with data reporting that the DHX29 (11227) mutant that only lacks the $\mathrm{OB}$ domain is inactive in the reconstituted system (Dhote et al. 2012). In conclusion, the eIF3-binding sites located at the $\mathrm{N}$ terminus and the ribosomal association site located at the C terminus of DHX29 are both important for the activity of the helicase during ribosomal scanning.

\section{DISCUSSION}

The initiation of translation in mammals is a complex and highly regulated process. General initiation relies on 


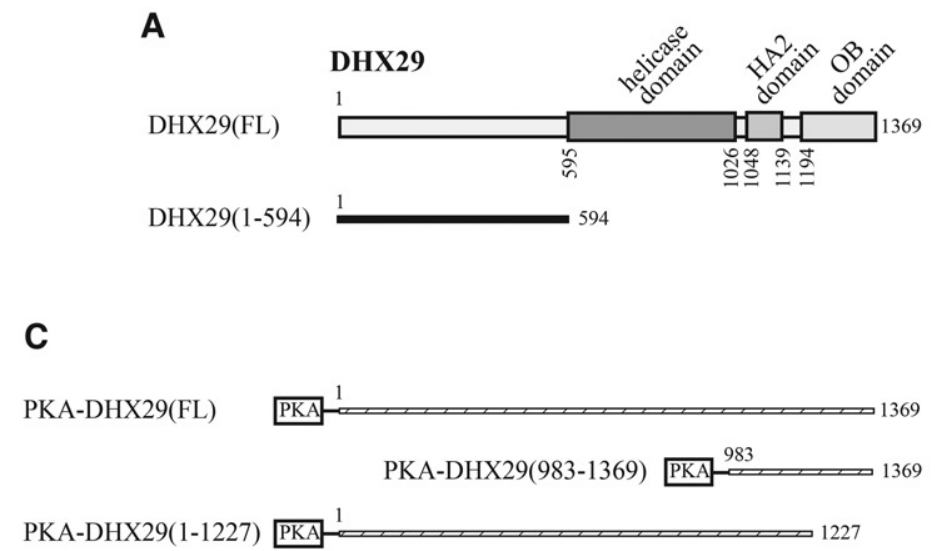

E

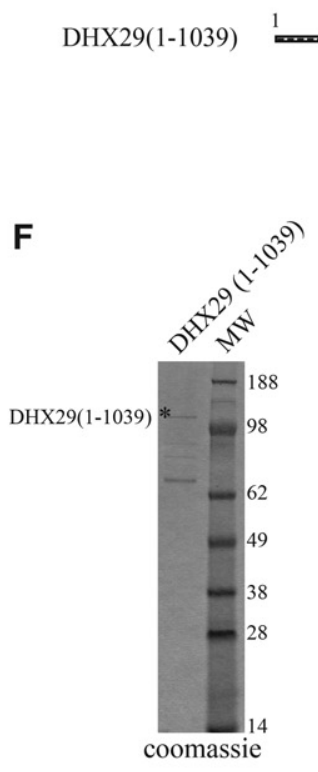

G

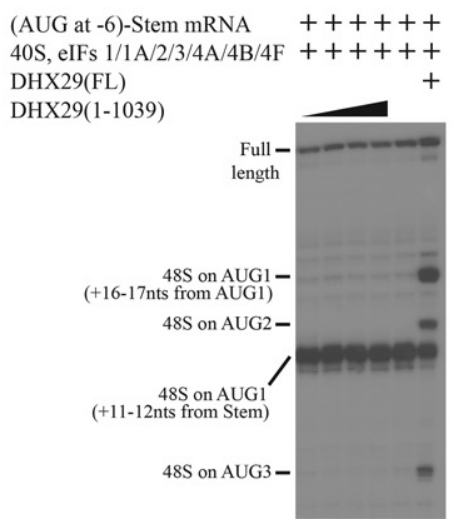

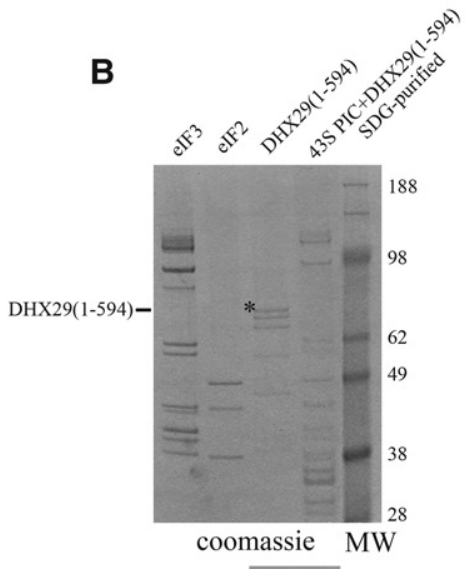

DHX29(1-594)-

anti-DHX29

D
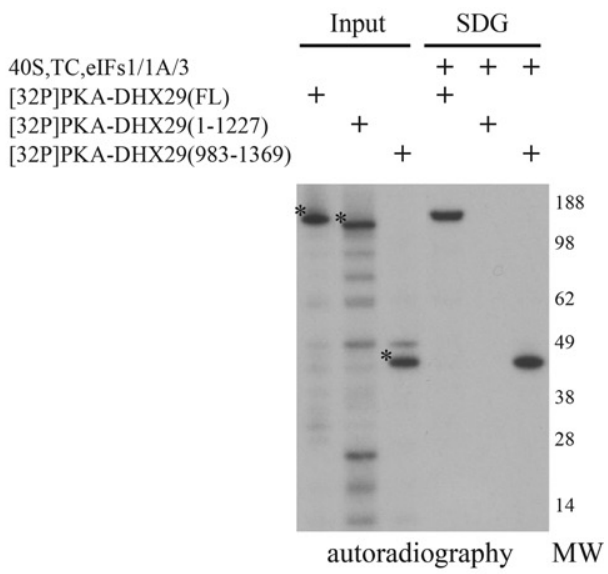

FIGURE 5. Association of different parts of DHX29 with the 43S PIC. Role of ribosomal binding in DHX29's activity during scanning. (A,C,E) DHX29 and its fragments used in the ribosomal binding assay. (B) Interaction between DHX29 (1-594) and the 43S PIC assayed by SDG centrifugation and SDS-PAGE of the peak ribosomal fraction followed by Coomassie blue staining and immunoblotting. The asterisk indicates the band of DHX29 (1-594). Positions of the molecular-weight markers are shown. (D) Association of different [ $\left.{ }^{32} \mathrm{P}\right]$-labeled forms of DHX29 with the 43S PIC analyzed by SDG centrifugation and SDS-PAGE of the peak ribosomal fraction followed by autoradiography. The asterisks indicate the main signals of the DHX29 proteins. (F) Purified recombinant DHX29 (1-1039) resolved by SDS-PAGE. The asterisk shows the band of DHX29 (1-1039). (G) Toeprint analysis of the formation of the 48S IC on (AUG at -6)-Stem mRNA in the presence of DHX29 (1-1039) at different concentrations and DHX29 (FL). Positions of the assembled 48S IC are indicated.

canonical initiation factors, whereas initiation on specific mRNAs requires different auxiliary proteins. DExH-box RNA helicase DHX29 was discovered to stimulate the formation of 48S ICs on mRNAs with structured 5'-UTRs in vitro, and its critical role in general translation was subsequently confirmed in vivo, thus identifying DHX29 as a canonical initiation factor. Moreover, the down-regulation of DHX29 inhibits cancer-cell growth in culture and in xenografts. Therefore, DHX29 could be considered a specific target in treatments for carcinogenesis. Because of the fundamental role of DHX29 in general translation and its relevance to human health, an understanding of the mechanism underlying its activity in initiation is critically important. Because DHX29 is not a processive helicase, it could either directly unwind the mRNA secondary structure during scanning or cause the remodeling of ribosomal complexes. To elucidate the mechanism, we used a reconstituted system coupled with targeted UV cross-linking and mutational analyses.

We found that DHX29 does not affect the mRNA path in the mRNA entry channel, and the pattern of cross-linked 
ribosomal proteins in the presence of the helicase also remains the same. Moreover, the helicase only weakly crosslinks to mRNA in this region. Considering the ribosomal position of DHX29 near the mRNA entrance, in the case of direct mRNA unwinding by helicase, the efficiency of mRNA cross-linking to a portion of the ribosomal proteins should decrease, whereas the efficiency of mRNA cross-linking to DHX29 should increase. Therefore, our data contradict the hypothesis on the direct mRNA unwinding activity of this protein (Pisareva et al. 2008). On the other hand, we detected some of the changes in cross-linking induced by DHX29 that support the mechanism of DHX29 activity via remodeling the architecture of ribosomal complexes. A cross-linking assay also detected that mRNA establishes contacts with several proteins in the context of $48 \mathrm{~S}$ IC as far as at the +15 position from the P-site.

A GST pull-down assay revealed that DHX29 establishes multiple contacts with eIF3 (Fig. 6). The unique $\mathrm{N}$ terminus of DHX29 contains at least two eIF3-binding sites. The first half of the DHX29 N terminus interacts with the eIF3a C terminus, whereas the second half associates with eIF3b RRM. The combination of the DHX29 helicase domain and C terminus also binds to eIF3. It is reported that this binding should occur at least at eIF3b and eIF3i.

Taking into account CryoEM data, we speculate that the $\mathrm{C}$ terminus of eIF3a, which is flexible and not resolved in the 43S PIC structure, has the potential to extend toward the A-site. The GST pull-down assay showed that the $\mathrm{C}$ terminus of eIF3a could establish a contact with the $\mathrm{N}$ terminus of DHX29 residing along the mRNA entry channel and extending up to the A-site. Interestingly, compared with the eIF3a in Saccharomyces cerevisiae, the eIF3a in mammals possesses a 418-aa C-terminal extension, although its function is not understood. Moreover, mRNAs with structured 5'-UTRs are more prevalent in mammals than in yeast because the greater complexity of higher eukaryote organization demands a more tightly regulated translation process. Therefore, both mammalian-specific DHX29 and the C-terminal extension of eIF3a could mediate scanning on structured mRNAs. It was recently reported that eIF3 binds to the 5'-UTRs of mRNAs involved in cell-growth control and regulates their

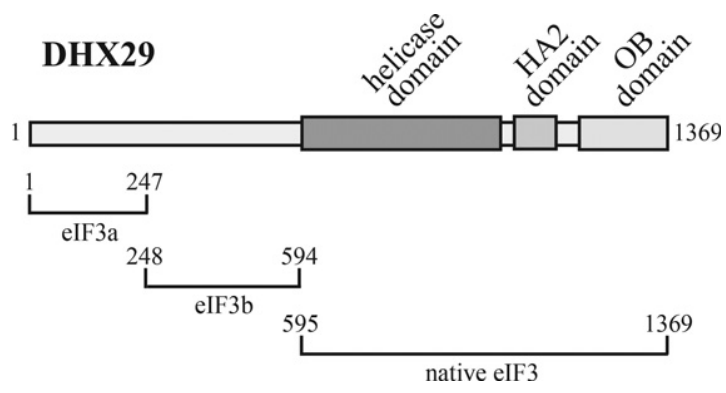

FIGURE 6. Diagram summarizing the interactions between different parts of DHX29 and subunits of eIF3. translation (Lee et al. 2015). This class of mRNAs is characterized by highly structured 5'-UTRs. Therefore, DHX29 could affect the interaction between mRNA and eIF3.

The deletion of the first half of the DHX29 N terminus completely abrogates the protein's activity in the unwinding of the mRNA secondary structure during scanning. The deletion of an 8-aa-long Q motif, that is located in the second half of the DHX29 $\mathrm{N}$ terminus and involved in the interaction with eIF3b RRM, also impairs protein activity. Moreover, we found that the N terminus of DHX29 is dispensable for ribosomal binding and the helicase associates with initiation complexes through its $\mathrm{C}$ terminus, and our results indicated that the $\mathrm{OB}$ domain is critical for binding. Taken together, our data confirm the importance of contacts between the DHX29 N terminus and eIF3 subunits for the unwinding of the mRNA secondary structure during scanning. Notably, contact between eIF3b RRM and the regulatory protein P311 was shown to be essential for the translation of transforming growth factors $\beta 1-3$ (Yue et al. 2014). Therefore, contact between eIF3b RRM and different accessory proteins is a determinant of various regulatory processes during translation.

In conclusion, we suggest the following mechanism for DHX29 activity during ribosomal scanning. Because DHX29 has a high affinity for both the 40S subunit and 43S PIC (Pisareva et al. 2008), it might bind the initiation complex at any step before the formation of the 48S IC. Upon ribosomal binding, DHX29 establishes contacts with different subunits of eIF3, thereby causing the rearrangement of the ribosomal complex. As a result, the remodeled ribosomal complex reveals higher processivity in the unwinding of the mRNA secondary structure during scanning.

\section{MATERIALS AND METHODS}

\section{Plasmids}

Expression vectors for His-tagged DHX29, eIF1, eIF1A, eIF4A, eIF4B, E. coli methionyl-tRNA synthetase, and GST-tagged eIF3b (FL and mutants) as well as transcription vectors for " +5 ," “ +7 ," " +9 ," and “+11" mRNAs, (AUG at -6)-Stem mRNA, and tRNA $_{i}{ }^{\text {Met }}$ have been previously described (Pisarev et al. 2008; Pisareva et al. 2008; Abaeva et al. 2011; Yue et al. 2014). Vectors for the E. coli-based expression of all His-tagged DHX29 deletion mutants were constructed by site-directed mutagenesis of a DHX29 expression vector. A His-tagged murine eIF3b (1-300) expression plasmid was prepared by inserting the corresponding ORF sequence into BamHI/XhoI sites of pET28b. PKA-DHX29 (FL), PKA-DHX29 (983-1369), and PKA-DHX29 (1-1227) expression vectors were produced by introducing the PKA site at the $\mathrm{N}$ terminus of the corresponding C-terminal His-tagged mutants. An expression vector for GST-tagged DHX29 (FL) was prepared by cloning the protein's coding region into BamHI/XhoI sites of pGEX-6P-1. Vectors for the expression of GST-tagged DHX29 deletion mutants were constructed by site-directed mutagenesis of GSTDHX29 (FL). GST-eIF3a (1-782) and GST-eIF3a (783-1382) expression plasmids were cloned by inserting the corresponding 
coding region of human eIF3a into SmaI/NotI and BamHI/NotI sites of pGEX-6P-1, respectively. Transcription vectors for the “+13" and "+15" mRNAs were produced by introducing cDNA following a T7 promoter into PstI/SmaI sites of pUC57 (GenScript). mRNAs and $\mathrm{tRNA}_{\mathrm{i}}{ }^{\text {Met }}$ were transcribed using T7 RNA polymerase. The (AUG at -6)-Stem mRNA transcript was capped with Vaccinia virus-capping enzyme (NEB) according to the manufacturer's protocol. Radiolabeled “ +5 ," “+7," “+9," “+11,” “+13,” and “+15” mRNAs containing 4-thioU were obtained by transcription in the presence of $\left[\alpha^{-32} \mathrm{P}\right]$ ATP and 4-thioU (TriLink Biotech).

\section{Antibodies}

We used DHX29, eIF3e, and T7tag antibodies (all from Bethyl Laboratories).

\section{Purification of translation components and aminoacylation of tRNA}

Native 40 S subunits, eIF2/3/4F, recombinant eIF1/1A/4A/4B, and $E$. coli methionyl-tRNA synthetase were purified as previously described (Pisareva et al. 2008). In vitro-transcribed $\mathrm{tRNA}_{i}{ }^{\mathrm{Met}}$ was aminoacylated with methionine in the presence of recombinant $E$. coli methionyl-tRNA synthetase as previously described (Pisareva et al. 2008).

\section{Purification of DHX29 WT and mutants}

Recombinant DHX29 (FL), DHX29 (25-1369), DHX29 (54-1369), DHX29 (248-1369), DHX29 (1-247), DHX29 (1-594), DHX29 (1-1039), and DHX29 $(\Delta \mathrm{Q})$ were expressed in $4 \mathrm{~L}$ of E. coli BL21 (DE3) media after induction with $0.1 \mathrm{mM}$ IPTG for $16 \mathrm{~h}$ at $16^{\circ} \mathrm{C}$. After expression, the proteins were isolated by affinity chromatography on Ni-NTA agarose followed by FPLC on MonoS (all forms of DHX29 except DHX29 [1-247] and DHX29 [1-594]) or monoQ (DHX29 [1-594]) columns. Because of the low ion-exchange properties, DHX29 (1-247) was not purified on FPLC, although it was used in the GST pull-down assay in the form of Ni-NTA eluate. FPLC fractions were collected across a $100-500 \mathrm{mM} \mathrm{KCl}$ gradient. DHX29 (FL), DHX29 (1-1039), DHX29 (248-1369), and DHX29 $(\Delta \mathrm{Q})$ were eluted at $310 \mathrm{mM}$; DHX29 (25-1369) and DHX29 (54-1369) were eluted at $140 \mathrm{mM}$; and DHX29 (1-594) was eluted at $320 \mathrm{mM} \mathrm{KCl}$.

Recombinant PKA-DHX29 (FL), PKA-DHX29 (983-1369), and PKA-DHX29 (1-1227) were expressed in 2 L of E. coli BL21(DE3) media after induction by $0.1 \mathrm{mM}$ IPTG for $16 \mathrm{~h}$ at $16^{\circ} \mathrm{C}$ and purified on Ni-NTA agarose according to the manufacturer's protocol.

\section{Purification of elF3b (1-300)}

Recombinant eIF3b (1-300) was expressed in 1 L of E. coli BL21 (DE3) media after induction by $1 \mathrm{mM}$ IPTG for $5 \mathrm{~h}$ at $37^{\circ} \mathrm{C}$ and isolated by affinity chromatography on Ni-NTA agarose followed by FPLC on a MonoQ column. Fractions were collected across a 100 $500 \mathrm{mM} \mathrm{KCl}$ gradient. eIF3b (1-300) was eluted at $330 \mathrm{mM} \mathrm{KCl}$.

\section{Lysate preparation of GST-tagged forms of DHX29, elF3a, and elF3b}

Recombinant GST-DHX29 (FL), GST-DHX29 (1-247), GST-DHX29 (248-594), GST-DHX29 (595-1369), GST-DHX29 (1-594), and GST-DHX29 (1-1039) as well as GST-eIF3b (FL), GST-eIF3b (1-500), GST-eIF3b (1-300), GST-eIF3b (1-160), GST-eIF3b (161-264), GST-eIF3a (1-782), and GST-eIF3a (7831382) were prepared in the form of E. coli lysates using an identical protocol. Each protein was expressed in $1 \mathrm{~L}$ of E. coli BL21(DE3) media after induction by $0.1 \mathrm{mM}$ IPTG for $16 \mathrm{~h}$ at $16^{\circ} \mathrm{C}$. The cells were collected, lysed in $20 \mathrm{~mL}$ buffer B (20 mM Tris- $\mathrm{HCl}, \mathrm{pH} 7.5$, $100 \mathrm{mM} \mathrm{KCl}, 0.1 \mathrm{mM}$ EDTA, 10\% glycerol) by sonication, clarified from cell debris by $10^{\prime} 15 \mathrm{krpm}$ centrifugation, and frozen at $-80^{\circ} \mathrm{C}$ for the downstream assay.

\section{Assembly of initiation complexes}

To reconstitute the eIF3/mRNA binary complex, 43S PIC and $48 \mathrm{~S}$ IC, we incubated different combinations of 0.2-2 pmol DHX29 (WT or mutants), $5 \mathrm{pmol}$ eIF2/3/4F, 7 pmol eIF1/1A/4A/4B, 5 pmol Met-tRNA ${ }_{i}^{\text {Met }}$, and 1.75 pmol $40 \mathrm{~S}$ ribosomal subunit in a $20 \mu \mathrm{L}$ reaction mixture containing buffer A $(20 \mathrm{mM}$ Tris- $\mathrm{HCl}, \mathrm{pH}$ 7.5, $100 \mathrm{mM} \mathrm{KCl}, 2.5 \mathrm{mM} \mathrm{MgCl}_{2}, 0.1 \mathrm{mM}$ EDTA, $1 \mathrm{mM}$ DTT) with $0.2 \mathrm{mM}$ ATP and $0.2 \mathrm{mM} \mathrm{GTP}$ for $10 \mathrm{~min}$ at $37^{\circ} \mathrm{C}$ in the presence of 0.3 pmol of the corresponding mRNA. In cross-linking experiments, we assembled the eIF3/mRNA complex and 48S IC on co-transcriptionally ${ }^{32} \mathrm{P}$-labeled and 4 -thioU-incorporated mRNAs.

\section{Toeprinting assay}

We estimated the yield and the mRNA position of the assembled $48 \mathrm{~S}$ IC via a toeprinting assay as previously described (Pisareva et al. 2008). This assay involves an extension by the reverse transcriptase of the $\left[{ }^{32} \mathrm{P}\right]$-labeled primer annealed to the mRNA. Generally, the 48 S IC yields a toeprint signal at the leading edge of the 40 S subunits at positions +16 to $+18 \mathrm{nt}$ downstream from the mRNA triplet in the P-site. The signals are visualized after electrophoresis of cDNA in the denatured polyacrylamide gel and autoradiography.

\section{UV-cross-linking assay}

To examine the effect of DHX29 on the pathway taken by mRNA in the initiation complexes, we used a UV-cross-linking assay. The $48 \mathrm{~S}$ ICs were reconstituted with/without DHX29 in an $80 \mu \mathrm{L}$ reaction mixture with scaled amounts of the initiation components on cotranscriptionally ${ }^{32} \mathrm{P}$-labeled and 4-thioU-incorporated " +5 ," “+7," “+9," “+11," “+13," and "+15” mRNAs. After assembly, the initiation complexes were purified from unbound components by centrifugation through $10 \%-30 \%$ SDG prepared in buffer $\mathrm{A}$ in a Beckman SW55 rotor at 53,000 rpm for $75 \mathrm{~min}$, irradiated at 360 $\mathrm{nm}$ for $30 \mathrm{~min}$ on ice using a UV Crosslinker (Spectroline), and digested with 5 units RNase A for $10 \mathrm{~min}$ at $37^{\circ} \mathrm{C}$. The samples were assayed by electrophoresis in NuPAGE $4 \%-12 \%$ Bis-Tris gel (Thermo Fisher Scientific) and autoradiography. After the RNase treatment of the samples, the cross-linked proteins acquired additional weight in the form of nucleotides, which resulted in a shifted band on SDS-PAGE. For the UV-cross-linking experiment with the 
eIF3/mRNA binary complex, we repeated this protocol but omitted the SDG purification step.

\section{GST pull-down assay}

To study the interaction between DHX29 and eIF3, we used a GST pull-down assay. A GST-tagged protein was preliminarily bound to Glutathione Sepharose 4B (GE Healthcare) and incubated with the purified recombinant partner; the complex was eluted with $1 \times$ Loading Buffer (Life Sciences), resolved using SDS-PAGE, and analyzed by immunoblotting.

\section{Ribosomal binding assay}

PKA-DHX29 (FL), PKA-DHX29 (983-1369), and PKA-DHX29 (1-1227) in Ni-NTA eluates were $\left[{ }^{32} \mathrm{P}\right]$-labeled with PKA enzyme (NEB) and $\left[\gamma^{-32} \mathrm{P}\right]$ ATP. To study the ribosomal association of different forms of DHX29, the 43S PICs assembled in a $400-\mu \mathrm{L}$ reaction mixture with scaled amounts of initiation components were supplemented with 50 pmol DXH29 (1-594) or $100 \mu \mathrm{L}$ Ni-NTA eluate containing $\left[{ }^{32} \mathrm{P}\right]$-labeled DHX29, purified from unbound components by centrifugation through 10\%-30\% SDG prepared in buffer A in a Beckman SW55 rotor at 53,000 rpm for $75 \mathrm{~min}$, resolved in SDS-PAGE, and analyzed by immunoblotting (DHX29 [1-594]) or autoradiography $\left(\left[{ }^{32} \mathrm{P}\right]\right.$-labeled forms of DHX29).

\section{RNA duplex-unwinding assay}

In this step, $2.5 \mu \mathrm{M} 5^{\prime}$-AAGUCCUCAG-3' RNA oligonucleotide was $\left[{ }^{32} \mathrm{P}\right]$-labeled with T4 PNK and annealed with an equimolar amount of complementary $5^{\prime}$-(CAAAA) ${ }_{5}$ CUGAGGACUU-3' RNA oligonucleotide, and the resulting RNA duplex was purified by gel filtration on a Spin-50 mini column (USA Scientific). Subsequently, $1 \mathrm{nM}$ purified duplex was incubated in a $10-\mu \mathrm{L}$ reaction mixture containing buffer A with $0.15 \mu \mathrm{M}$ DHX29, DHX29/ eIF3, or eIF4A/eIF4F and $0.2 \mathrm{mM} \mathrm{ATP}$ at $37^{\circ} \mathrm{C}$ for $40 \mathrm{~min}$. The reaction was stopped, and the samples were assayed by $12 \%$ nondenatured PAGE and autoradiography as previously described (Pisareva et al. 2008).

\section{ACKNOWLEDGMENTS}

We thank Tatyana Pestova for the (AUG at -6)-Stem mRNA transcription vector, Anton Komar for the DHX29 expression vector, and Lucia Schuger for her gift of GST-tagged eIF3b expression constructs. This work was supported by the National Institutes of Health (GM097014 to A.V.P.).

Received June 7, 2016; accepted September 12, 2016.

\section{REFERENCES}

Abaeva IS, Marintchev A, Pisareva VP, Hellen CUT, Pestova TV. 2011. Bypassing of stems versus linear base-by-base inspection of mammalian mRNAs during ribosomal scanning. EMBO J 30: $115-129$.

Asano K. 2014. Why is start codon selection so precise in eukaryotes? Translation (Austin) 2: e28387.
Asano K, Vornlocher HP, Richter-Cook NJ, Merrick WC Hinnebusch AG, Hershey JW. 1997. Structure of cDNAs encoding human eukaryotic initiation factor 3 subunits. Possible roles in RNA binding and macromolecular assembly. J Biol Chem 272: 27042-27052.

Asano K, Phan L, Anderson J, Hinnebusch AG. 1998. Complex formation by all five homologues of mammalian translation initiation factor 3 subunits from yeast Saccharomyces cerevisiae. J Biol Chem 273: $18573-18585$.

Aylett CHS, Boehringer D, Erzberger JP, Schaefer T, Ban N. 2015. Structure of a yeast 40S-eIF1-eIF1A-eIF3-eIF3j initiation complex. Nat Struct Mol Biol 22: 269-271.

Chiu W-L, Wagner S, Herrmannová A, Burela L, Zhang F, Saini AK, Valásek L, Hinnebusch AG. 2010. The C-terminal region of eukaryotic translation initiation factor $3 \mathrm{a}$ (eIF3a) promotes mRNA recruitment, scanning, and, together with eIF3j and the eIF3b RNA recognition motif, selection of AUG start codons. Mol Cell Biol 30: $4415-4434$.

Cuchalová L, Kouba T, Herrmannová A, Danyi I, Chiu W-L, Valásek L. 2010. The RNA recognition motif of eukaryotic translation initiation factor $3 \mathrm{~g}$ (eIF3g) is required for resumption of scanning of posttermination ribosome for reinitiation on GCN4 and together with eIF3i stimulates linear scanning. Mol Cell Biol 30: 4671-4686.

des Georges A, Dhote V, Kuhn L, Hellen CUT, Pestova TV, Frank J, Hashem Y. 2015. Structure of mammalian eIF3 in the context of the $43 S$ preinitiation complex. Nature 525: 491-495.

Dhote V, Sweeney TR, Kim N, Hellen CUT, Pestova TV. 2012. Roles of individual domains in the function of DHX29, an essential factor required for translation of structured mammalian mRNAs. Proc Natl Acad Sci 109: E3150-E3159.

Elantak L, Wagner S, Herrmannová A, Karásková M, Rutkai E, Lukavsky PJ, Valásek L. 2010. The indispensable N-terminal half of eIF3j/HCR1 cooperates with its structurally conserved binding partner eIF3b/PRT1-RRM and with eIF1A in stringent AUG selection. J Mol Biol 396: 1097-1116.

Enchev RI, Schreiber A, Beuron F, Morris EP. 2010. Structural insights into the COP9 signalosome and its common architecture with the $26 \mathrm{~S}$ proteasome lid and eIF3. Structure 18: 518-527.

Harms U, Andreou AZ, Gubaev A, Klostermeier D. 2014. eIF4B, eIF4G and RNA regulate eIF4A activity in translation initiation by modulating the eIF4A conformational cycle. Nucleic Acids Res 42: 7911-7922.

Hashem Y, des Georges A, Dhote V, Langlois R, Liao HY, Grassucci RA, Hellen CUT, Pestova TV, Frank J. 2013. Structure of the mammalian ribosomal $43 \mathrm{~S}$ preinitiation complex bound to the scanning factor DHX29. Cell 153: 1108-1119.

Hinnebusch AG, Lorsch JR. 2012. The mechanism of eukaryotic translation initiation: new insights and challenges. Cold Spring Harb Perspect Biol 4: a011544.

Jackson RJ, Hellen CUT, Pestova TV. 2010. The mechanism of eukaryotic translation initiation and principles of its regulation. Nat Rev Mol Cell Biol 11: 113-127.

Lee ASY, Kranzusch PJ, Cate JHD. 2015. eIF3 targets cell-proliferation messenger RNAs for translational activation or repression. Nature 522: 111-114.

Linder P, Jankowsky E. 2011. From unwinding to clamping - the DEAD box RNA helicase family. Nat Rev Mol Cell Biol 12: 505-516.

Parsyan A, Shahbazian D, Martineau Y, Petroulakis E, Alain T, Larsson O, Mathonnet G, Tettweiler G, Hellen CU, Pestova TV, et al. 2009. The helicase protein DHX29 promotes translation initiation, cell proliferation, and tumorigenesis. Proc Natl Acad Sci 106: 22217-22222.

Pena V, Liu S, Bujnicki JM, Lührmann R, Wahl MC. 2007. Structure of a multipartite protein-protein interaction domain in splicing factor prp8 and its link to retinitis pigmentosa. Mol Cell 25: 615-624.

Phan L, Zhang X, Asano K, Anderson J, Vornlocher HP, Greenberg JR, Qin J, Hinnebusch AG. 1998. Identification of a translation initiation 
factor 3 (eIF3) core complex, conserved in yeast and mammals, that interacts with eIF5. Mol Cell Biol 18: 4935-4946.

Pisarev AV, Kolupaeva VG, Yusupov MM, Hellen CUT, Pestova TV. 2008. Ribosomal position and contacts of mRNA in eukaryotic translation initiation complexes. EMBO J 27: 1609-1621.

Pisareva VP, Pisarev AV. 2016. DHX29 reduces leaky scanning through an upstream AUG codon regardless of its nucleotide context. Nucleic Acids Res 44: 4252-4265.

Pisareva VP, Pisarev AV, Komar AA, Hellen CUT, Pestova TV. 2008. Translation initiation on mammalian mRNAs with structured 5'UTRs requires DExH-box protein DHX29. Cell 135: $1237-1250$.
Schaefer MH, Wanker EE, Andrade-Navarro MA. 2012. Evolution and function of CAG/polyglutamine repeats in protein-protein interaction networks. Nucleic Acids Res 40: 4273-4287.

Valásek L, Phan L, Schoenfeld LW, Valásková V, Hinnebusch AG. 2001. Related eIF3 subunits TIF32 and HCR1 interact with an RNA recognition motif in PRT1 required for eIF3 integrity and ribosome binding. EMBO J 20: 891-904.

Yue MM, Lv K, Meredith SC, Martindale JL, Gorospe M, Schuger L. 2014. Novel RNA-binding protein P311 binds eukaryotic translation initiation factor 3 subunit $b$ (eIF3b) to promote translation of transforming growth factor $\beta 1-3$ (TGF- $\beta 1-3)$. J Biol Chem 289: 33971-33983. 

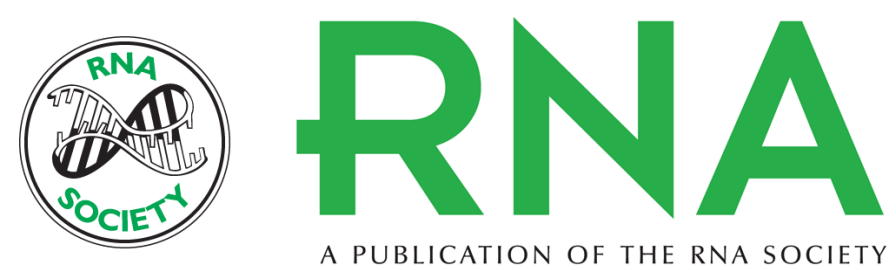

\title{
DHX29 and elF3 cooperate in ribosomal scanning on structured mRNAs during translation initiation
}

\author{
Vera P. Pisareva and Andrey V. Pisarev
}

RNA 2016 22: 1859-1870 originally published online October 12, 2016

Access the most recent version at doi:10.1261/rna.057851.116

\begin{abstract}
References This article cites 26 articles, 12 of which can be accessed free at: http://rnajournal.cshlp.org/content/22/12/1859.full.html\#ref-list-1

Creative This article is distributed exclusively by the RNA Society for the first 12 months after the Commons full-issue publication date (see http://rnajournal.cshlp.org/site/misc/terms.xhtml). After 12 License months, it is available under a Creative Commons License (Attribution-NonCommercial 4.0 International), as described at http://creativecommons.org/licenses/by-nc/4.0/.
\end{abstract}

Email Alerting Receive free email alerts when new articles cite this article - sign up in the box at the Service top right corner of the article or click here. 